\title{
Alternativas educativas para la optimización de la expresión oral en estudiantes de secundaria
}

\author{
Educational alternatives for the optimization of oral expression in secondary school students
}

Estratégia didática para a otimização da expressão oral em alunos do ensino médio

\section{Ronald Estefano Sanabria Coronado}

sanabriacoronado@outlook.es

https://orcid.org/0000-0002-3856-4165

\section{RESUMEN}

En el ambiente educativo la expresión oral es una competencia comunicativa de gran importancia ya que permite comprender los mensajes que se emiten durante el desarrollo del proceso de enseñanza y aprendizaje. Sin embargo, los estudiantes de secundaria presentan deficiencias en su expresión oral, que se evidencia al realizar exposiciones didácticas. Por esta razón esta investigación tuvo como objetivo diseñar una estrategia didáctica para la optimización de la expresión oral. La población estuvo conformada por 20 estudiantes que cursan el cuarto de secundaria y dos de sus docentes de la Unidad Educativa Nuevo Tiempo en Potosí, Bolivia. Es una investigación proyectiva, con diseño de campo y mixto. Las técnicas de recolección de los datos fueron la observación no participativa, la encuesta y la entrevista y los instrumentos estuvieron constituidos por: una guía estructurada de observación, una guía de entrevista y un cuestionario. Se diseñó una propuesta fundamentada en principios psicológicos, didácticos, pedagógicos y de la comunicación.

Palabras clave: Expresión oral; competencia comunicativa; estrategia didáctica; estudiantes de secundaria; comunicación verbal
ABSTRACT

In the educational environment, oral expression is a communicative competence of great importance since it allows us to understand the messages that are emitted during the development of the teaching and learning process. However, high school students present deficiencies in their oral expression, which is evidenced by making didactic presentations. For this reason, this research aims to design a didactic strategy for the optimization of oral expression. The population was made up of 20 students who are in the fourth year of secondary school and two of their teachers from the Nuevo Tiempo Educational Unit in Potosí, Bolivia. It is a projective investigation, with field and mixed design. The data collection techniques were non-participatory observation, the survey and the interview, and the instruments consisted of: a structured observation guide, an interview guide, and a questionnaire. A proposal based on psychological, didactic, pedagogical and communication principles was designed.

Key words:

Oral expression, communicative competence, didactic strategy, high school students, verbal communication
RESUMO

No ambiente educacional, a expressão oral é uma competência comunicativa de grande importância, pois permite compreender as mensagens que são emitidas durante o desenvolvimento do processo de ensino e aprendizagem. No entanto, alunos do ensino médio apresentam deficiências na expressão oral, o que é evidenciado por meio de apresentações didáticas. Por esse motivo, esta pesquisa visa traçar uma estratégia didática para a otimização da expressão oral. A população foi composta por 20 alunos do quarto ano do ensino médio e dois de seus professores da Unidade Educacional Nuevo Tiempo de Potosí, Bolívia. É uma investigação projetiva, com campo e desenho misto. As técnicas de coleta de dados foram a observação não participante, a pesquisa e a entrevista, e os instrumentos foram constituídos por: roteiro de observação estruturado, roteiro de entrevista e questionário. Foi desenhada uma proposta baseada em princípios psicológicos, didáticos, pedagógicos e de comunicação.

Palavras-chave:

Expressão oral; competência comunicativa; estratégia didática; alunos do ensino médio; Comunicação verbal 


\section{INTRODUCCIÓN}

El lenguaje es una de las habilidades que caracteriza al ser humano, y le permite la comunicación de sus ideas para desenvolverse en sociedad. La correcta expresión oral consiste en la formulación de las ideas de forma clara, precisa y coherente constituyendo una importante competencia comunicativa, junto a la lectura y a la escritura.

En el mundo actual son numerosos los contextos en los cuales es importante el desarrollo de la expresión oral, uno de esos contextos es el educativo; no se trata de enseñar a hablar a los jóvenes, porque eso lo hacen desde muy pequeños, el propósito de atender la expresión oral en el ámbito educativo es para el beneficio de los estudiantes no sólo en su aprendizaje sino también, en su autoestima y seguridad.

Uno de los problemas que en la actualidad preocupa a los profesionales en el área de la educación, es la expresión oral, porque existe poca participación en el aula por parte de los estudiantes, así mismo se observan deficiencias en la expresión oral; como consecuencia, surgen dificultades en el proceso de enseñanza aprendizaje y en la comunicación en el aula, por ello se considera que uno de los aspectos psicopedagógicos más relevantes para atender, es el fortalecimiento de la expresión oral en la etapa de la escolaridad.

La expresión oral es un constructo teórico complejo, por lo cual recibe diferentes definiciones al respecto Baralo 2000 señala que es:

Una destreza o habilidad de comunicación que no tiene sentido sin la comprensión, sin el procesamiento y la interpretación de lo escuchado. La expresión oral implica la interacción y la bidireccionalidad, en un contexto compartido, y en una situación en la que se deben negociar los significados ( $p$. 164).
La bidireccionalidad implica un emisor que envía el mensaje y un receptor que capta el mensaje y lo procesa, elabora una respuesta que se convierte en un nuevo mensaje. Martin Barbero (2002) y Rodríguez (2006) consideran que la comunicación no se debe entender solo en dos direcciones, sino que es un fenómeno complejo y por lo tanto multidireccional, varias personas como emisores y receptores emitiendo su opinión, y además el mensaje no sólo son las palabras sino también, el tono, el volumen, la intención y la kinestesia o lenguaje corporal.

Es importante resaltar que la comunicación multidireccional tiene mayor relevancia en el proceso de enseñanza - aprendizaje, porque los estudiantes se comunican con el docente y con sus compañeros, de tal forma que la dinámica del grupo enriquece las posibilidades de aprendizaje significativo, para ello debe generarse una participación activa y autónoma para conseguir el correcto flujo de información.

En este mismo orden de ideas Ramírez (2002) agrega que la expresión oral consiste en prestar atención y ser receptivo a los signos que puedan servir para interpretar el mensaje, hablar el mismo lenguaje no solo se refiere al idioma sino a todo aquello favorezcan la riqueza comunicativa para procesar y comprender los mensajes.

Cervantes (2016) resume que la expresión oral es la destreza lingüística relacionada con la producción del discurso oral. Es una capacidad comunicativa que abarca no solo un dominio de la pronunciación del léxico y la gramática de la lengua, si no también unos conocimientos socioculturales y pragmáticos. Consta de una serie de micro destrezas, tales como saber aportar información y opiniones, mostrar acuerdo o desacuerdos, resolver fallos conversacionales o saber en qué circunstancias es pertinente hablar y en cual no. 
Estos autores tienen diferentes aportes para la definición de la expresión oral, en síntesis se entiende como una destreza comunicativa que está a acompañada de múltiples mensajes no solo verbales sino también corporales, culturales y otros propios del grupo en el cual se realiza la comunicación; tiene como propósito captar, procesar, interpretar y comprender los mensajes.

Para Hernández (2011) los elementos que componen la expresión corporal y que deben ser considerados cuando se va a diseñar una propuesta para desarrollarla son: la dicción, la fluidez, el volumen, la intensidad, el tono, el ritmo, vocabulario pertinente y el orden lógico de las ideas que conforman el mensaje.

En el aula de clases las exposiciones académicas a cargo de los estudiantes son una estrategia utilizada con regularidad para evaluar sus conocimientos, esta actividad exige que el expositor tenga una adecuada expresión oral.

Según Verderber (2010) la exposición académica es aquella presentación oral que se realiza en un contexto académico con la principal intención de dar a conocer un tema específico siguiendo un orden introducción, desarrollo y conclusión, utilizando un lenguaje formal, sencillo y comprensible a la audiencia.

El expositor cumple con la función de informar o explicar acerca de un tema, debe presentar las ideas de forma ordenada y progresiva, responder a las preguntas y generar participación en la audiencia, todos estos requisitos son difíciles de conseguir en estudiantes de secundaria sino han tenido una formación en expresión oral.

Con base en la observación previa y a su experiencia en la Unidad Educativa Nuevo Tiempo el investigador identifica, en los estudiantes de cuarto de secundaria, las siguientes dificultades en relación con la expresión oral durante el desarrollo de las exposiciones didácticas: (a) Dificultad para expresar o comunicar un mensaje de forma coherente y claro, (b) Se muestran inseguros, tartamudean, usan muletillas, se quedan en silencio, (c) Carencias en el vocabulario para expresar sus ideas y hacerse comprender por otras personas, (d) Poca capacidad para escuchar con atención y mostrar respeto por un expositor, (e) Vocabulario insuficiente e impreciso, (f) Postura corporal inadecuada (g) Inadecuado uso del volumen, tono e intensidad de la voz.

Con base en esta realidad y la importancia de la expresión oral para el desenvolvimiento académico en la actualidad y en el futuro esta investigación se plantea como interrogante de investigación ¿Cuáles son los elementos de una estrategia didáctica que permita optimizar la expresión oral de los estudiantes de cuarto de secundaria de la Unidad Educativa Nuevo Tiempo en Potosí, Bolivia?

Y el objetivo norte del estudio fue: Diseñar una estrategia didáctica para optimizar la expresión oral en estudiantes de cuarto de secundaria de la Unidad Educativa Nuevo Tiempo del municipio de Potosí en Bolivia.

\section{MÉTODO}

La investigación fue proyectiva ya que culmina con el diseño una estrategia didáctica para atender las necesidades diagnosticadas. El enfoque es mixto porque se recolectan y analizan datos cualitativos y cuantitativos. El diseño es de campo no experimental pues los datos se recolectaron en el contexto natural.

La población estuvo conformada por 20 estudiantes, con edades comprendidas entre los 14 y 15 años de edad que cursan el cuarto de secundaria en la unidad educativa Nuevo Tiempo, ubicada en la zona de San Martín del municipio de Potosí en Bolivia; también dos docentes que atienden este grupo estudiantes desde hace cuatro años. 
Las técnicas empleadas para la recolección de los datos fueron la observación no participativa, la encuesta y la entrevista.

Los instrumentos estuvieron constituidos por: una guía estructurada de observación, una guía de entrevista y un cuestionario. La guía de observación, con 11 ítems permitió realizar la observación de la expresión oral de los estudiantes en las clases de música, biología y sociales, así como también en actividades extra catedra, para un total de
8 horas de observación. La guía de entrevista semiestructurada con 11 preguntas para medir las dimensiones de la expresión oral, se aplicó a los docentes y el cuestionario de 11 ítems se aplicó a los estudiantes para conocer desde su percepción la expresión oral de sus compañeros de clase.

La variable expresión oral se operacionalizó para la construcción de los instrumentos con dos dimensiones: Habilidad expresiva y habilidad cognitiva (Ver Cuadro 1).

Cuadro 1. Operacionalización de la variable.

\begin{tabular}{|c|c|c|c|c|c|}
\hline \multirow[b]{2}{*}{ Definición operativa } & \multirow[b]{2}{*}{ Dimensiones } & \multirow[b]{2}{*}{ Indicadores } & \multicolumn{3}{|c|}{ Instrumentos } \\
\hline & & & 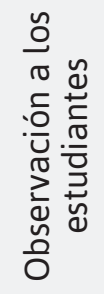 & 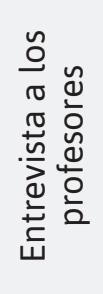 & 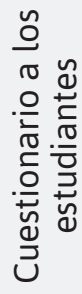 \\
\hline \multirow{10}{*}{$\begin{array}{l}\text { La expresión oral es una habilidad } \\
\text { para transmitir información a } \\
\text { partir de la interacción en un } \\
\text { contexto compartido y que no } \\
\text { tiene sentido sin la comprensión, } \\
\text { sin el procesamiento y } \\
\text { la interpretación de lo escuchado. }\end{array}$} & \multirow[t]{7}{*}{$\begin{array}{l}\text { Habilidad } \\
\text { expresiva }\end{array}$} & $\begin{array}{l}\text { Explica términos complejos que } \\
\text { utiliza en su discurso }\end{array}$ & $x$ & $x$ & $x$ \\
\hline & & Se expresa con fluidez & $x$ & $x$ & $x$ \\
\hline & & $\begin{array}{l}\text { Eleva el volumen de voz en } \\
\text { palabras importantes }\end{array}$ & $x$ & $x$ & $x$ \\
\hline & & $\begin{array}{l}\text { Intercala el ritmo entre lento y } \\
\text { rápido }\end{array}$ & $x$ & $x$ & $x$ \\
\hline & & $\begin{array}{l}\text { Tonalidad de voz acorde al } \\
\text { ambiente }\end{array}$ & $x$ & $x$ & $x$ \\
\hline & & $\begin{array}{l}\text { Vocabulario comprensible y } \\
\text { sencillo }\end{array}$ & $x$ & $\mathrm{x}$ & $x$ \\
\hline & & Genera dinámicas participativas & $x$ & $x$ & $x$ \\
\hline & \multirow[t]{3}{*}{$\begin{array}{l}\text { Habilidad } \\
\text { cognitiva }\end{array}$} & $\begin{array}{l}\text { Crea un argumento } \\
\text { coherente con inicio, desarrollo y } \\
\text { final }\end{array}$ & $x$ & $x$ & $x$ \\
\hline & & Responde preguntas & $x$ & $\mathrm{x}$ & $x$ \\
\hline & & Explica de diferentes formas & $x$ & $x$ & $x$ \\
\hline
\end{tabular}




\section{RESULTADOS}

Se presentan los resultados de acuerdo a la información recolectada con cada instrumento.

\section{Guía de observación}

La guía de observación mide las dos dimensiones de la expresión oral: habilidad expresiva y habilidad cognitiva.

Los resultados de la habilidad expresiva se resumen en el Gráfico 1.

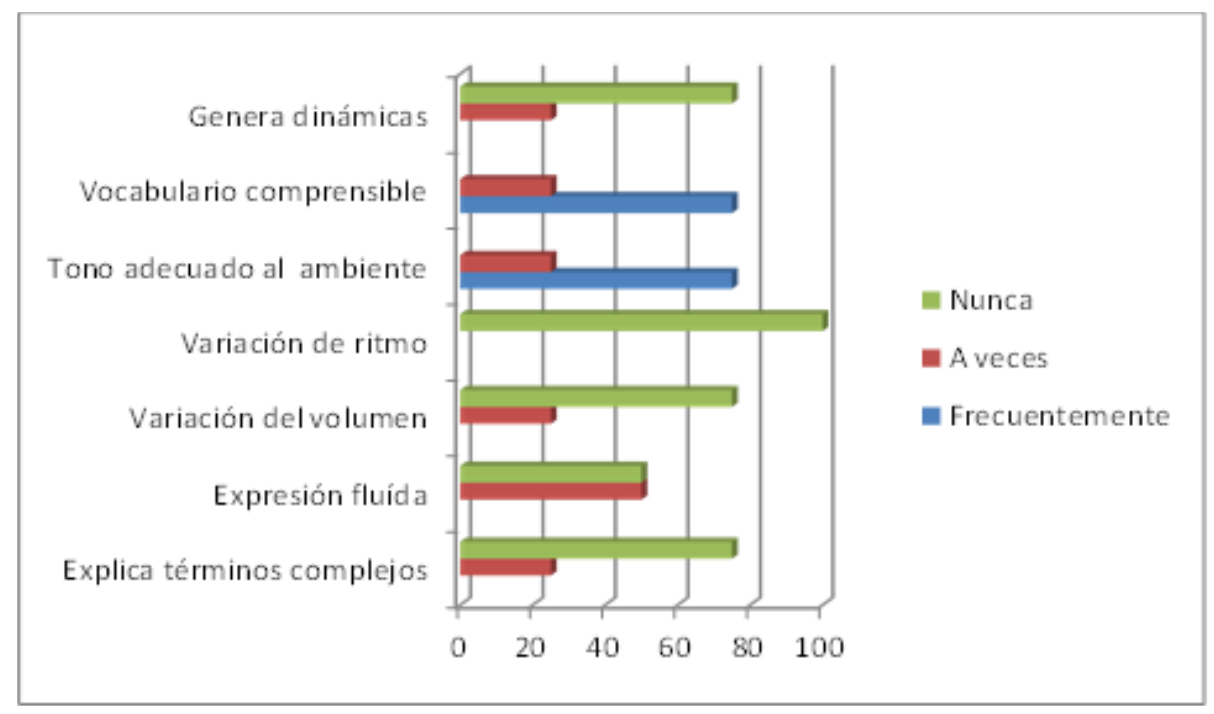

Gráfico 1. Habilidad expresiva.

El $75 \%$ de los estudiantes adecuan el tono de voz de acuerdo al lugar en el cual se realiza la exposición, tanto si es en un ambiente abierto o cerrado. También el $75 \%$ de los individuos mostraron un vocabulario comprensible y sencillo en el desarrollo de las clases y en las exposiciones. Estas dos características son una fortaleza del grupo en cuanto a su expresión oral ya que el tono de voz y utilizar un lenguaje comprensible permite una buena comunicación.

Por otra parte, ninguno de los estudiantes observados controla el ritmo del discurso, mantienen más bien un ritmo rápido, esta característica afecta la comprensión del contenido que se está desarrollando en la exposición.
También se identificaron debilidades en tres indicadores, el 75\% de los individuos no elevaron el volumen de la voz para enfatizar palabras o ideas importantes, tampoco explicaron el significado de los términos complejos en su discurso o exposición y no generaron dinámicas participativas para incluir a los oyentes en la comunicación.

Estos resultados evidencian una habilidad expresiva baja lo que impide que el mensaje sea comprendido.

Los resultados de la habilidad cognitiva se resumen en el Gráfico 2. 


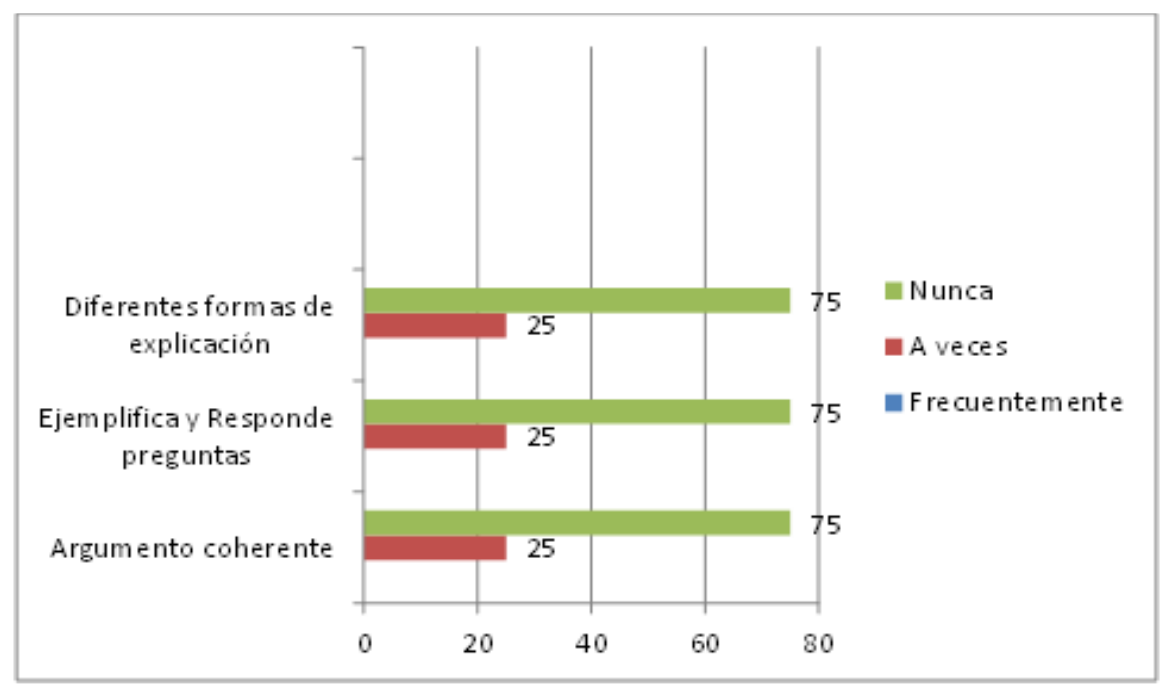

Gráfico 2. Habilidad cognitiva.

La capacidad de crear argumentos de forma coherente con principio, desarrollo y fin es esencial para que haya una comunicación efectiva. De acuerdo a los resultados es una debilidad en este grupo de estudio, el $75 \%$ de los estudiantes nunca fueron capaces de crear dicha coherencia en el proceso de una exposición, se pudo evidenciar que al momento de exponer no desarrollaron adecuadamente el orden de la exposición. Una exposición debe permitir la comunicación efectiva de un contenido, para ello es necesario que el mensaje cuente con todas sus etapas para tener coherencia.

El $75 \%$ de los estudiantes nunca explican de diferentes formas para ser mejor comprendidos debido a que la explicación que utilizan es memorística y pocos son los que investigan a profundidad lo que tienen que exponer.

El $75 \%$ de los estudiantes jamás realiza la ejemplificación para ser mejor entendido debido a que las explicaciones que realizan son pocos los que tienen conocimiento propio de lo que explican.

La comprensión del tema es fundamental para tener una excelente expresión oral para exponer el contenido en cualquier asignatura.

\section{Entrevista a los docentes}

Se pregunta a los docentes como una fuente de información adicional a las observaciones. Los dos docentes entrevistados coinciden que los estudiantes cuando realizan exposiciones acerca de un tema presentan dificultades, algunas de ellas son que: no realizan preguntas acerca de términos o conceptos, el expositor no responde comprensivamente las preguntas que surgen, las exposiciones no tienen un hilo conductor coherente de inicio, desarrollo y cierre del tema; por lo general los estudiantes muestran poco interés en realizar exposiciones porque si se equivocan son objeto de burlas por parte de sus compañeros.

Según los docentes se debe atender la formación de estrategias en expresión oral y sobre todo se debe trabajar en crear un clima de confianza para que los estudiantes se sientan seguros al realizar sus exposiciones.

\section{Cuestionario a los estudiantes}

Este instrumento tiene como objetivo la evaluación de los estudiantes por sus compañeros de clase. Las preguntas están en relación con el desempeño de otros. (Ver Gráfico 3.) 


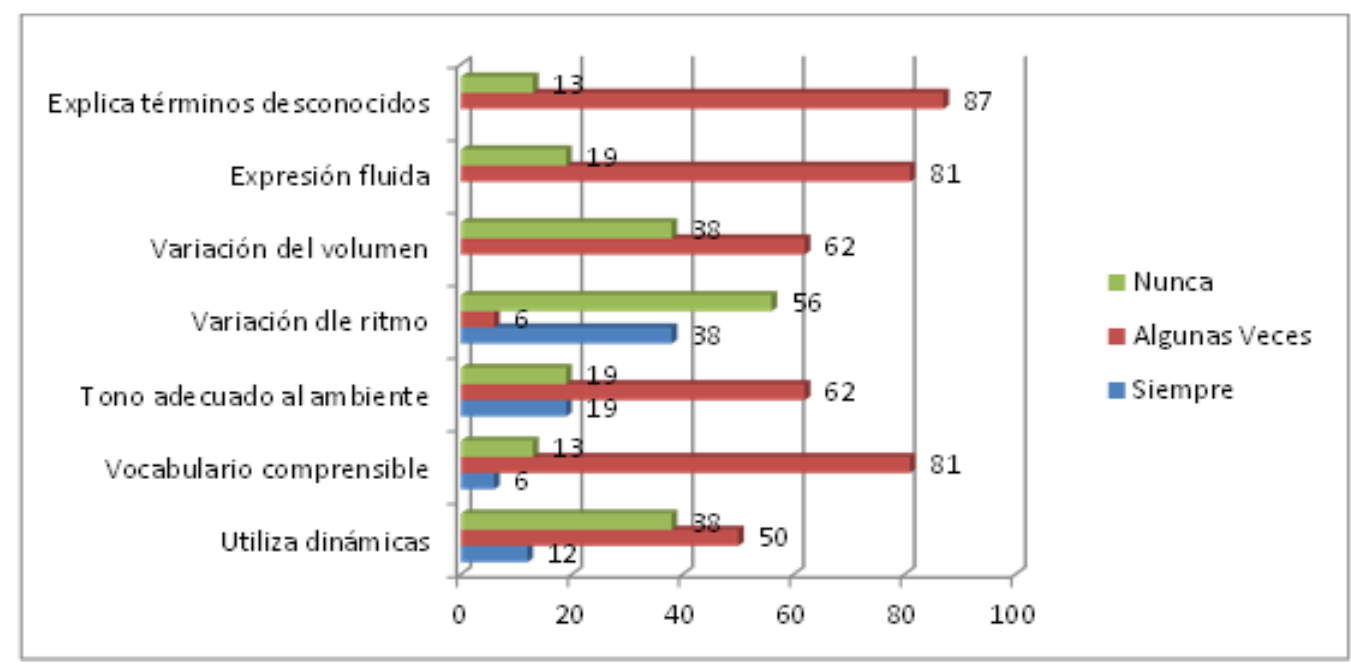

Gráfico 3. Dimensión: habilidad Expresiva percepción de los estudiantes.

El indicador relacionado con la explicación de términos desconocidos, el $87 \%$ de los estudiantes respondió que sus compañeros de estudio, cuando realizan exposiciones, a veces explican términos desconocidos y el $13 \%$ consideran que nunca hacen explicación de términos desconocidos.

Estos resultados demuestran que existe poca habilidad al momento de expresarse oralmente, debido a que para que exista una buena expresión oral es necesario poder ser entender lo que se expresa, porque la expresión oral no simplemente es saber expresar, sino también ser entendido por los demás.

El indicador relacionado con la fluidez durante la exposición, el $81 \%$ de los estudiantes considera que sus compañeros a veces se expresan de manera fluida, y $19 \%$ opinan que nunca las exposiciones se realizan con fluidez.

La fluidez es una de las características específicas de la expresión oral porque tiene que existir un ritmo o intercambio de velocidad para transmitir algún mensaje y hacer que la comunicación sea efectiva.

Para el indicador relacionado con la utilización de un vocabulario comprensible y sencillo al momento de exponer un $81 \%$ de los estudiantes respondió que a veces lo utilizan pero en ocasiones los expositores se aprenden palabras de memoria que no conocen su significado y esto hace poco comprensible la exposición.

El vocabulario comprensible es una característica importante para la expresión oral, porque mejora la interpretación y favorece a la comunicación.

Acerca del indicador relacionado con el volumen de voz en palabras importantes para centrar la atención de sus compañeros, mencionan que el $63 \%$ de los estudiantes a veces hacen uso de cambios en el volumen de la voz en palabras importantes.

Para el cambio del tono de voz en lugares abiertos respondieron que un $63 \%$ de los estudiantes a veces cambian el tono de voz en las ferias que se realizan en el patio del establecimiento debido al nerviosismo ante la atención de los profesores. El tono de voz es una de las características de las habilidades expresivas, esto está en función a mejorar la comprensión al momento de exponer en diferentes lugares, por tal motivo el cambio en el tono de voz es importante para expresar las ideas de un tema en particular.

El Gráfico 4 muestra los resultados obtenidos de la segunda dimensión, las habilidades cognitivas, desde la percepción que tienen los estudiantes del desempeño de sus compañeros de clase durante las exposiciones. 


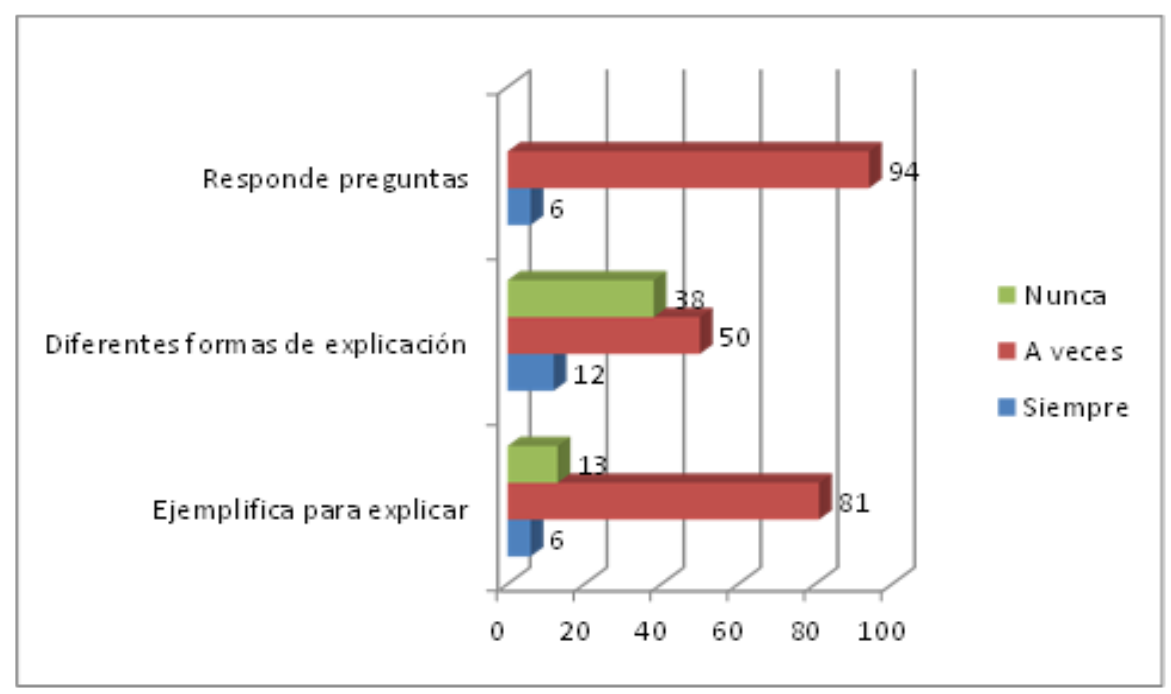

Gráfico 4. Dimensión: habilidad Cognitiva percepción de los estudiantes.

El $81 \%$ de los estudiantes perciben que sus compañeros a veces utilizan ejemplos para explicar el contenido, el $13 \%$ considera que nunca lo hacen, solo un $6 \%$ perciben que se siempre se usan ejemplos.

Considerando el uso de diferentes formas de explicar un contenido el $50 \%$ declara que ocurre algunas veces, para el $38 \%$ nunca se usan diferentes formas de explicación y el $12 \%$ percibe que siempre se explica de diferentes formas dentro de la exposición de sus compañeros de estudio.

El ítem si se le da respuesta a la preguntas realizadas el $94 \%$ dice que a veces y el $6 \%$ siempre.

Triangulando los resultados desde el investigador, los docentes y los propios estudiantes de cuarto de secundaria, se evidencia que las fortalezas que presentan los estudiantes en relación con su expresión oral son el tono de voz acorde con el ambiente en el cual se desarrolla la exposición y el vocabulario comprensible y sencillo. Asimismo las mayores debilidades diagnosticadas son la explicación de términos complejos, elevación del volumen de voz en palabras importantes, la fluidez al momento de exponer sobre algún tema en particular, la realización de dinámicas durante una exposición.
Según los resultados se puede afirmar que el $75 \%$ de los estudiantes se encuentran con déficit y bajo desarrollo de la expresión oral, declaran intranquilidad y falta de confianza cuando tienen que realizar exposiciones y agregan que no conocen la importancia de la expresión y su influencia en el aprendizaje.

Con base en los resultados del diagnóstico se plantea a continuación la propuesta para atender estas carencias y debilidades.

\section{Propuesta “Crea, expresa y aprende”}

\section{Justificación}

La propuesta fundamenta sus actividades en los principios de la didáctica, la pedagogía y la psicología educativa. El principio de ordenamiento de la estructura didáctica como inicio, desarrollo y cierre; así como también la mayor diversificación de actividades en diferentes asignaturas, de tal forma que cambia el contenido pero se ejercitan los elementos de la expresión oral y la comunicación. En el marco de la pedagogía se organiza el desarrollo de habilidades desde las más simples, básicas hacia las más complejas, el uso de la tecnología para aumentar la motivación 
de acuerdo a las características e intereses del grupo. La psicología educativa aporta la necesidad de mantener la creatividad, la participación y la colaboración para lograr los mejores resultados.

\section{Usuarios y contexto}

Está dirigido a estudiantes de cuarto de secundaria de la Unidad Educativa Nuevo Tiempo, del municipio Potosí en Bolivia. La propuesta se desarrollará en el ambiente escolar habitual, adicionalmente los docentes pueden adecuar espacios apropiados para el desarrollo de las actividades en los cuales los estudiantes se puedan desarrollar las actividades.

\section{Objetivos}

- Optimizar la expresión oral en estudiantes de cuarto de secundaria

- Mejorar la comunicación efectiva en el aula de los estudiantes de cuarto de secundaria.

- Fomentar el aprendizaje significativo para solventar el bajo rendimiento.

\section{Recursos y materiales}

- Audiovisuales: equipos de computación, videos, micrófonos, parlantes, programas, equipos de proyección.
- Impresos: libros, revistas, afiches, periódicos, trípticos, fotografías, mapas, láminas didácticas.

- Otros: materiales específicos para las actividades como: estambre, marcadores, reglas, cartulinas, láminas de papel, lápices, pelotas, cuerdas, globos.

- Evaluación: Se contempla una evaluación continua para reconocer los cambios que se susciten en cada participante y de forma grupal.

\section{Estructura}

La propuesta está constituida por cinco talleres, cinco debates y una "Feria de Evaluación" dirigidos a los estudiantes. También dos talleres para los docentes con el propósito de actualizar algunos elementos importantes de la expresión oral y motivarlos a la participación en la ejecución de la propuesta.

Los talleres son cinco y la estructura de cada taller es: nombre del taller, duración, responsable, participantes, objetivo, recursos, actividades de inicio, desarrollo y cierre y actividad de evaluación. A continuación se resume la propuesta planificada para los estudiantes. 
Taller 1: Expresión Oral

Duración: 30 minutos

Responsable: Facilitador

Participantes: 20 estudiantes de cuarto de secundaria

Objetivo: Orientar la adaptación de la expresión oral para su aplicación en el discurso de los estudiantes durante una exposición.

Recursos: Ovillo de lana, cartulinas, marcadores, data display.

\section{Momentos}

Momento de inicio: Se desarrolla la dinámica denominada la telaraña que tiene como objetivo generar un clima de confianza entre los participantes.

La Telaraña

Se forma un circulo, con todos los participantes sentados estrechamente uno con otro, además se les explica en qué consiste la dinámica, posteriormente se lanza el ovillo de lana a uno de los participantes quien responde con su nombre y apellido y comparte algún comentario de algún interés o actividad que practica. Se asegura el hilo del ovillo en su mano y lanza el ovillo a otro participante, quien realizará la misma acción que el anterior. Así se forma una telaraña, al terminar el ultimo participante deberá deshacerse siguiendo el mismo procedimiento, pero ahora mencionar a los participantes como algo positivo sobre la personal al que le regresa el otro extremo y así continuar hasta llegar a la primera persona que tiene la punta de la lana. Tendrá una duración de 15 minutos.

Momento de desarrollo: Se explica el contenido de la temática: la expresión oral, su definición, características, su importancia, papel de la expresión oral en la escuela, ventajas de la expresión oral, factores que impiden la correcta expresión oral. Esto tendrá una duración de 20 minutos. La exposición debe ser participativa, con ejemplos y la intervención de los estudiantes a través de la realización de preguntas activadoras.

Momento de cierre: En este momento del desarrollo se motivara a los estudiantes a partir de los aspectos positivos que conlleve una buena expresión oral.

Evaluación: Para culminar con el primer taller se realizara la dinámica del dado que permita evaluar la comprensión. El Dado

Todos los participantes forman un circulo, el organizador deberá entrega unas fichas con números del 1 al 6 de manera que todos puedan tener su respectivo número, uno de los participantes lanza el dado, se hace una pregunta y la responde uno de los estudiantes que tenga el numeró que salió en el dado. Sin son varios estudiantes con el mismo número se reúnen y deciden la respuesta. Las preguntas están en relación con el tema desarrollado.

El organizador deberá anotar las respuestas emitidas por los estudiantes para valorar el nivel de comprensión del taller. Duración: 10 minutos. 
Taller 2. Cuña publicitaria de radio

Duración: 45 minutos

Responsable: Facilitador

Participantes: 20 estudiantes de cuarto de secundaria

Objetivo: Fortalecer en los estudiantes la fluidez de la voz para una mejor expresión oral.

Recursos: Micrófonos, radios y grabadores de audio.

\section{Momentos}

Momento de inicio: Desarrollo de la dinámica llamada Punto Loco para crear un clima de confianza en los estudiantes antes del desarrollo del taller.

Punto Loco

Se forman cuatro grupos compuestos de cinco personas, el facilitador solicitará de forma general, que los grupos puedan sostenerse ya sean de 10, 5, o 4 puntos en donde los participantes deberán realizar esta acción sin soltarse uno de otro, es importante explicar que los puntos solo pueden ser las puntas de los pies, rodillas y dedos de las manos. Duración de 5 minutos.

Momento de desarrollo: Se explica el contenido de la temática, con una participación activa, con ejemplos y la intervención de los estudiantes a través de la realización de preguntas activadoras. El contenido es la función que cumple la fluidez de la voz en la expresión oral y la comunicación efectiva.

Se escucharan diferentes cuñas publicitarias para su análisis y caracterización. Duración: 20 minutos

Momento de cierre: Culminar con el taller se desarrolla la dinámica denominada cuña radiofónica que permita poner en práctica la explicación de la temática planteada.

Cuña Radiofónica

Los estudiantes forman 4 grupos, cada grupo diseña y graba una cuña publicitaria para radio para campañas de sensibilización, prevención, venta de productos o servicios para un consumidor. 20 minutos.

Evaluación: Guía de observación no participativa para el registro de la actividad. 
Taller 3. Cuenta cuentos

Duración: 45 minutos

Responsable: Facilitador

Participantes: 20 estudiantes de cuarto de secundaria

Objetivo: Generar en los estudiantes habilidades expresivas a través de la creatividad del cuenta cuentos.

Recursos: Cuentos, poesías, historias.

\section{Momentos}

Momento de inicio: Desarrollo de la dinámica llamada "el rumor o corre el chisme" para crear un clima de confianza en los estudiantes antes del desarrollo del taller.

El Rumor

El organizador forma un círculo con todos los participantes y será parte del mismo. Comienza el facilitador contando una información al oído del participante que se encuentre a su derecha. Cada participante irá pasando la información sin cambiarla. Así hasta concluir con el último participante del círculo que dirá en voz alta el comentario que le llegó. El facilitador lee el comentario inicial y se comparan las dos informaciones. Duración de 5 minutos.

Momento de desarrollo: Se explica el contenido de la temática, con una participación activa, con ejemplos y la intervención de los estudiantes a través de la realización de preguntas activadoras. El contenido es la velocidad de la expresión oral, el volumen o tono de voz adecuado para cada ambiente y su relación con otras características de la comunicación verbal. Se ejemplifica la técnica de contar cuentos, la importancia de dar distintas entonaciones y tonos de la voz para asegurar una correcta emisión del mensaje. Duración: 20 minutos.

Momento de cierre: Para culminar con el taller se realiza la dinámica denominada: "El cuenta cuentos" en la cual los estudiantes desarrollan un cuento de acuerdo a su creatividad.

\section{El Cuentacuentos}

El facilitador forma un círculo y explica a los participantes que van a contar un cuento entre todos, para esto deberán nombrar a uno de los participantes para que pueda copiar el cuento, también puede ser grabado. Posteriormente un participante al azar comienza el cuento con una frase cualquiera y así cada uno va aportando una idea al cuento en construcción.

Se lee el cuento que se produjo en grupo, se puede complementar con la lectura de una noticia o historia de forma dramatizada. Duración: 20 minutos.

Se les solicita a los estudiantes su impresión acerca de las actividades realizadas. Duración: 20 minutos.

Evaluación: Guía de observación no participativa para el registro de la actividad. 


\section{Taller 4. El valor de la comunicación en la familia y en la sociedad}

Duración: 50 minutos

Responsable: Facilitador

Participantes: 20 estudiantes de cuarto de secundaria

Objetivo: Orientar a los estudiantes acerca de la importancia de la adquisición de herramientas para una buena comunicación en los entornos: educativos, familiares y sociales.

Recursos: Reproductor de audio, data display, computadora, papel bond y marcadores.

\section{Momentos}

Momento de inicio: Desarrollo de la dinámica llamada "el viaje des estresante" para activar la atención, concentración y generar participación de los estudiantes antes del desarrollo del taller.

Viaje des estresante

El organizador forma un círculo con todos los participantes, les pide a los participantes que cierren sus ojos y se concentren en los sonidos que van a escuchar. El facilitador reproduce audios de relajación, con música o sonidos de la naturaleza. También les pide a los participantes que se concentren en su respiración, que inhalen y exhalen profundamente, luego de tres minutos se les pedirá que abren los ojos. Duración de 5 minutos.

Momento de desarrollo: Proyección de un video con relación a la comunicación humana, posteriormente se trabajaran los contenidos acerca de la importancia de la comunicación en el hogar y en la sociedad y como interviene la expresión oral en la comunicación y las micro destrezas que contribuyen a la toma de decisiones y resolución de conflictos. Duración: 20 minutos.

Momento de cierre: El facilitador planteará un caso problema de interacción social, los estudiantes se reunirán en grupo para analizar el planteamiento y proporcionar diferentes soluciones. Resumir el análisis y la solución en un papel para exponer a todo el grupo en plenaria. Deben identificar elementos de la expresión oral que favorecen la comunicación y minimizan las situaciones problemas.

Se les solicita a los estudiantes su impresión acerca de las actividades realizadas. Duración: 20 minutos.

Evaluación: Guía de observación no participativa para el registro de la actividad. 


\section{Debate 1. El castigo físico de los padres a los hijos ¿Los corrige o los trauma?}

Objetivo: Favorecer en los estudiantes la práctica del diálogo, análisis y el razonamiento para la construcción de un discurso con argumento lógico.

Participantes: Participantes: 20 estudiantes de cuarto de secundaria.

\section{Momentos}

Momento de inicio: Explicación en qué consiste la técnica del debate, el tiempo en el cual desarrolla, las normas; los tipos de debate de acuerdo a su objetivo, el rol del moderador, los resultados. Se promueve la participación activa del estudiante. Antes de la actividad se puede asignar una lectura que aborde la temática desde diferentes visiones: psicológico, legal, social.

Momento de desarrollo: Se forman 4 equipos, se plantean las normas del debate, se asigna un moderador, un secretario, cada equipo tendrá 10 minutos para discutir en el grupo acerca de los aspectos de la temática, beneficios o perjuicios del castigo. Una vez culminado el tiempo, el facilitador solicita que se formen dos equipos uno argumenta que el castigo corrige y el otro equipo argumenta que el castigo traumatiza, se ejecuta el debate y se llega a un resultado o veredicto.

Momento de cierre: El facilitador solicita la culminación del debate, ambas partes deberán llegar a un acuerdo. Terminado el debate se pregunta a los estudiantes sus sensaciones y percepciones acerca de la importancia de la argumentación coherente.

Evaluación: Guía de observación no participativa para el registro de la actividad.

\section{Debate 2. Legalización del aborto ¿Acuerdo o desacuerdo?}

Objetivo: Favorecer en los estudiantes la práctica del diálogo, análisis y el razonamiento para la construcción de un discurso con argumento lógico.

Participantes: Participantes: 20 estudiantes de cuarto de secundaria.

\section{Momentos}

Momento de inicio: Repaso para recordar en qué consiste la técnica del debate, el tiempo en el cual desarrolla, las normas; los tipos de debate de acuerdo a su objetivo, el rol del moderador, los resultados. Se promueve la participación activa del estudiante. Antes de la actividad se puede ver un video acerca del aborto y sus connotaciones psicológicas, educativas, económicas y salud.

Momento de desarrollo: Se forman 4 equipos, se plantean las normas del debate, se asigna un moderador, un secretario, cada equipo tendrá 10 minutos para discutir en el grupo acerca de los aspectos de la temática, a favor o en contra. Una vez culminado el tiempo, el facilitador solicita que se formen dos equipos un equipo argumenta a favor del aborto y el otro equipo argumenta en contra, se ejecuta el debate y se llega a un resultado o veredicto.

Momento de cierre: El facilitador solicita la culminación del debate, ambas partes deberán llegar a un acuerdo. Terminado el debate se pregunta a los estudiantes sus sensaciones y percepciones acerca de la importancia de la argumentación coherente.

Evaluación: Guía de observación no participativa para el registro de la actividad. 
Evaluación de la Propuesta "Crea, expresa y aprende":

Al finalizar la ejecución de la propuesta se realiza una feria evaluativa en la cual todos los involucrados tienen la oportunidad de emitir sus opiniones acerca del impacto de las actividades realizadas para la optimización de la expresión oral de los estudiantes de cuarto año de educación secundaria. Esta información se registra en un cuestionario semiestructurado y en una guía de entrevistas no estructuradas.

\section{CONCLUSIONES}

Los estudiantes del cuarto año de educación secundaria de la unidad educativa Nuevo Tiempo del municipio de Potosí presentan una limitada expresión oral al realizar las exposiciones en clases, con dificultades en las habilidades expresivas y cognitivas. Las mayores debilidades se encontraron en la ausencia de estrategias para construir un argumento lógico, la dificultad para mantener un tono y volumen de voz que mantenga el interés de la ausencia, el uso de ejemplos para lograr la comprensión del discurso, la explicación de términos de difícil comprensión. Los estudiantes asumen que deben tener la responsabilidad de preparar la temática con responsabilidad.

Desde la perspectiva de los docentes y de los estudiantes es necesaria la formación en expresión oral de forma sistemática y no asumir que los estudiantes aprenden estas habilidades al cumplir las actividades asignadas por sus profesores.

La estrategia didáctica para atender la expresión oral está fundamentada en principios psicológicos, pedagógicos y didácticos. Se estructuró en talleres y debates que se conciben para ejecutarlos en cuatro bimestres, un largo período del tiempo escolar, para lograr el desarrollo de la expresión oral.

La estructura de la estrategia didáctica está compuesta por los siguientes elementos: el nombre de la estrategia, objetivos, recursos, medios, contexto, aplicación y evaluación. La ejecución consta de tres momentos: inicio, desarrollo y cierre.

\section{REFERENCIAS}

Baralo, M. (2000). El desarrollo de la expresión oral en el aula. Revista Nebrija de Lingüística Aplicada a la Enseñanza de lengua 164. https:// cvc.cervantes.es/ensenanza/biblioteca_ele/ carabela/pdf/47/47_005.pdf

Cervantes 2016 Cervantes. (1 de noviembre de 2016). Centro Virtual Cervantes. http://www. cvc.cervantes.es

Hernández Gutiérrez, M. (2011). Técnicas de expresión oral. San Gil.: SENA Centro Agro turístico

Martín-Barbero, J. (2002). La educación desde la comunicación. México: Gustavo Gili

Ramírez Martínez, J. (2002). La expresión oral. La Rioja: Contextos Educativos

Rodríguez Luna, M. (2006). Consideraciones sobre el discurso oral en el aula. Bogotá, Colombia. Universidad Distrital Francisco José De Caldas. Revista Enunciación. http://www.udistrital.edu. $\mathrm{co} /$

Verderber, R. (2010). Desarrollo y exposición de presentaciones grupales. Comunicación oral efectiva. Trad. Jorge Manzano Olmos México D.F.: CENGAGE Learning 